\title{
ARC MODELING IN INDUSTRIAL APPLICATIONS
}

\author{
Ch. RÜmpler ${ }^{a, *}$, R. Chechare $^{b}$, A. Zacharias ${ }^{a}$ \\ ${ }^{a}$ Eaton Industries GmbH, Hein-Moeller-Straße 7-11, 53115 Bonn, Germany \\ ${ }^{b}$ Eaton India Innovation Center, Magarpatta SEZ Tower B6, Pune, India-411013 \\ * ChristianRuempler@Eaton.com
}

\begin{abstract}
Simulation methods are routinely applied in the design and development process of power distribution devices. Arcing phenomena that occur during switching operations or fault events are modeled to optimize device performance and gain deeper insights into the behavior that testing cannot easily provide. In this contribution, some applications are presented in detail. The first example describes the distribution of debris that is generated inside a molded case circuit breaker (MCCB) during short-circuit interruption. A model is used to analyze the debris transport and to derive a solution to address issues caused by the debris. Second application example is a cooling device for hot plasma gases vented by circuit breakers. A model driven design process helps to define the device dimensions to achieve a safe temperature level of the exhaust gases. The third example deals with short-circuit behavior of a hollow core high voltage surge arrester, comparing model and experimental results.
\end{abstract}

Keywords: arc interruption modeling, molded case circuit breaker, debris transport, blow-out cooling, surge arrester.

\section{Introduction}

Modeling methods have been developed to predict the arcing behavior in power distribution devices and are nowadays applied on a regular basis in various research and development activities. A major goal of any modeling activity in industrial applications is towards the risk mitigation of a design. By means of modeling and simulation, major processes that influence the design performance should be represented by the model, although in many cases the physical processes involving arcing are still too complex to include every aspect. But with feasible assumptions, useful models can be developed that provide guidance in the design process or enable a better understanding of the arcing phenomena and interactions. With appropriate utilization of these models, one can improve the designs ahead of testing and go into the test laboratory with higher confidence to achieve successful results.

In this contribution we introduce a basic modeling approach in Sec. 2 and provide three different applications in the following sections where the modeling approach was applied. The first example in Sec. 3 describes the distribution of debris that is generated inside a low-voltage molded case circuit breaker (MCCB) during short-circuit interruption. Based on arc model results, particle tracking is used to predict the debris transport inside the breaker and to derive a solution to address issues caused by the debris. The second application example in Sec. 4 deals with a cooling device for hot plasma gases that are vented by circuit breakers during short-circuit interruption. A model driven design process helps to define the gas cooling device dimensions and to achieve a safe temperature level of the exhaust gases, in order to reduce the risks that are involved with hot and potentially still ionized venting gas. The third example in Sec. 5 describes the shortcircuit behavior of a hollow core high voltage surge arrester and modeling thereof. Model results as well as experimental results are provided and compared.

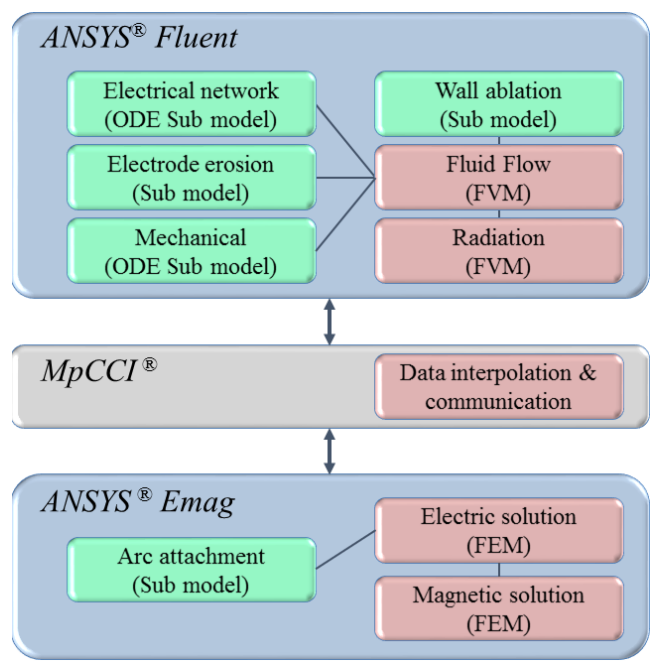

Figure 1. Arc simulation framework.

\section{General arc modeling approach}

In all application examples, as given in the following sections, the arc is a thermal plasma [1] where a magneto-hydrodynamic (MHD) modeling approach can be applied. Partial differential equations for fluid flow (Navier-Stokes), electromagnetic fields (Maxwell), as well as radiation transport are solved in a partitioned code-coupling approach [2]. With help of the 
commercial solver $A N S Y S^{\circledR}$ Fluent [3], fluid and radiation equations are solved using finite volume methods. The electric and magnetic field equations are solved by $A N S Y S^{\circledR}$ Emag solver, which is based on the finite element theory. The necessary data exchange between these solvers is done by the code coupling tool $M p C C I^{\circledR}$ [4], as illustrated in Fig. 1. Besides the built-in functionality of the solvers, additional modules have been implemented to represent more interactions and processes in the model, which is needed to achieve a useful accuracy of the model results:

- ordinary differential equation solver (ODE solver) to describe the interaction of the arc with the electrical network,

- electrode erosion model to determine the metal vapor that is injected into the fluid region [2],

- wall ablation model for plastic materials [5],

- ODE solver for prediction of contact arm motion (driven by magnetic forces or the mechanism of a breaker),

- arc attachment model [2].

For achieving best computational performance we use the built-in parallelization capabilities of the solvers. In addition to that, we benefit in this setup from an explicit coupling approach where the codes are running in parallel as well [2], maximizing the utilization of computational resources.

\section{Application: Debris reduction for low-voltage MCCB}

\subsection{Introduction}

Short-circuit (SC) interruption testing of low-voltage molded case circuit breakers (MCCB's) has to be performed e.g. according to IEC standard 60947-2 [6]. An example for a low-voltage MCCB design with the arc chamber in focus is depicted in Fig. 2. When testing with very high prospective currents (depending on the targeted interruption ratings of the device), the current has to be limited quickly and interrupted successfully. Even with effective current limiting by means of a quick contact opening, fast arc formation, and fast rising arc voltage, the let-through currents can still be in the range of tens of kilo-amperes. High current densities flowing through the arc attachment zones can cause erosion of the electrode materials in the form of liquid droplets that are splashing away or generate metal vapor. This is happening while the arc attaches to the contacts (various contact materials), to the contact arm (usually copper), or the splitter plates (usually ferromagnetic steel).

The gas inside the arc chamber heats up quickly and the arc chamber is pressurized due to energy dissipation inside the arc. Venting openings behind the splitter plates define the main flow path from the interior of the breaker to the surroundings, while some flow is also going in the other direction towards

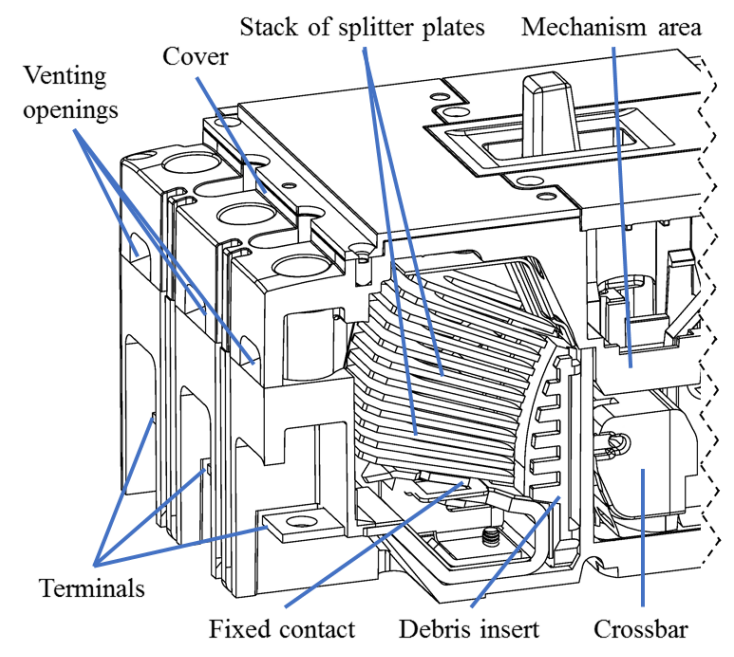

Figure 2. Low-voltage circuit breaker with debris catching insert [7].

the mechanism that is used to operate the crossbar and the contacts. Due to this venting path, liquid or already solidified metal droplets or particles are transported towards the crossbar and mechanism area of the breaker, potentially causing issues due to interference with moving parts. To better understand the behavior, arc modeling combined with particle tracking is performed. Based on the results, a novel design has been developed and successfully tested, as presented in the next subsections.

\subsection{Arc model}

The modeling approach, as described in Sec. 2, is used to investigate the arc behavior and flow conditions during a $70 \mathrm{kA} / 415 \mathrm{~V} / 60 \mathrm{~Hz}$ short-circuit interruption of a low-voltage MCCB that has a rated current of $225 \mathrm{~A}$. Only a single pole of the breaker is modeled and a symmetry boundary condition at the center of the pole is used to reduce the numerical effort. Model results that show the arc location and engagement with the contacts as well as the splitter plates, are depicted in Fig. 3 for two time points corresponding to smaller and larger contact opening angle.

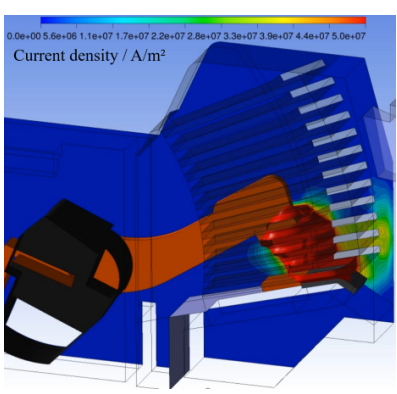

(a) $\mathrm{t}=1.0 \mathrm{~ms}$

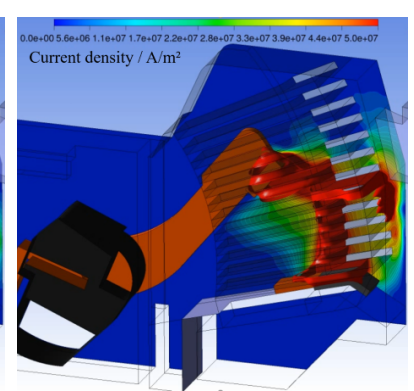

(b) $\mathrm{t}=1.5 \mathrm{~ms}$
Figure 3. Arc model results showing iso-surface of current density at $5 \cdot 10^{7} \mathrm{~A} / \mathrm{m}^{2}$ for two time points. 


\subsection{Debris transport modeling}

Based on the arc model results, particle motion inside the arc chamber can be investigated. Using the calculated flow field at selected time points (frozen flow field), particle tracking is performed. Although the particles might interact with the flow in reality and change the flow conditions somehow, this approach is still able to provide an indication how the molten droplets will distribute inside the arc chamber. To define the source of the particles in the model, the arc attachment areas on any metal part - like contacts or splitter plates - are used, where the current density exceeds a threshold value. As the droplet dimensions are varying, some assumptions have to be made in the model. Droplet diameters of $0.5 \mathrm{~mm}$ and $1 \mathrm{~mm}$ are used in this study, where results are very similar for both diameters. Fig. 4 shows calculated particle tracks for $0.5 \mathrm{~mm}$ particle diameter at two different time points (corresponding to the arc model results depicted in Fig. 3). Once particles in the model are released and moving due to the flow, they can hit solid parts (e.g. arc chamber walls, splitter plates). In this case we use an escape boundary condition, so that the particle is removed from the calculation. With this assumption, we are focusing on the main particle transport paths where the particles can move without interferences.

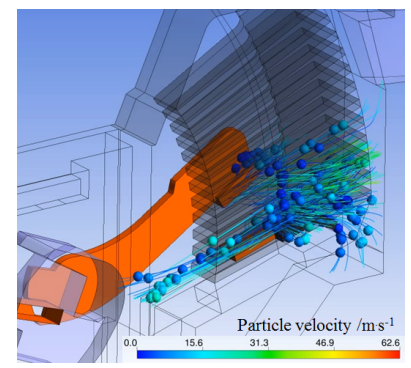

(a) $\mathrm{t}=1.0 \mathrm{~ms}$

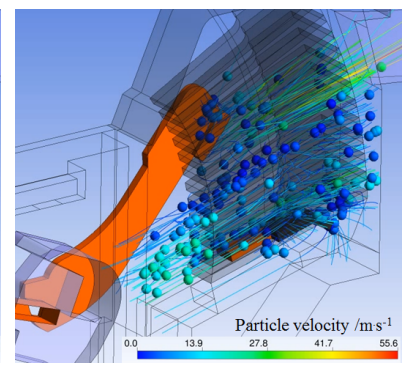

(b) $\mathrm{t}=1.5 \mathrm{~ms}$
Figure 4. Visualization of modeled particle transport for two different time points.

Because the arc is attaching on both contacts and some splitter plates at both time points, particles are generated there. Due to the strong gas stream away from the contacts towards the vent opening, majority of particles are transported into this direction. At 1.0 ms many particles hit the arc chamber wall behind the splitter plates and are removed from calculation there. But due to a recirculation zone inside the stack of splitter plates, particles are transported towards the rear of the breaker (towards the contact arm pivot point or mechanism area). The same effect is visible in the results for time point $1.5 \mathrm{~ms}$. With larger contact opening angle, more particles find a free path towards the venting opening, but the recirculation pattern in the splitter plate stack transfers particles to the rear side of the breaker. As described above, this can cause issues with the mechanical operation of the breaker.

\subsection{Solution for debris reduction}

The erosion of the contacts or splitter plates cannot be avoided, therefore a method to reduce the impact of debris is needed. In order to avoid debris coming into the crossbar or mechanism area, the recirculation flow pattern can be utilized as shown in Fig. 5. By placing an insert into the arc chamber behind the splitter plate stack, as shown in the picture, the particles that are transferred backwards with the gas stream will end up in a pocket where they can accumulate. Fig. 2 shows the placing of the insert into the arc chamber as well as the pockets to accumulate debris.

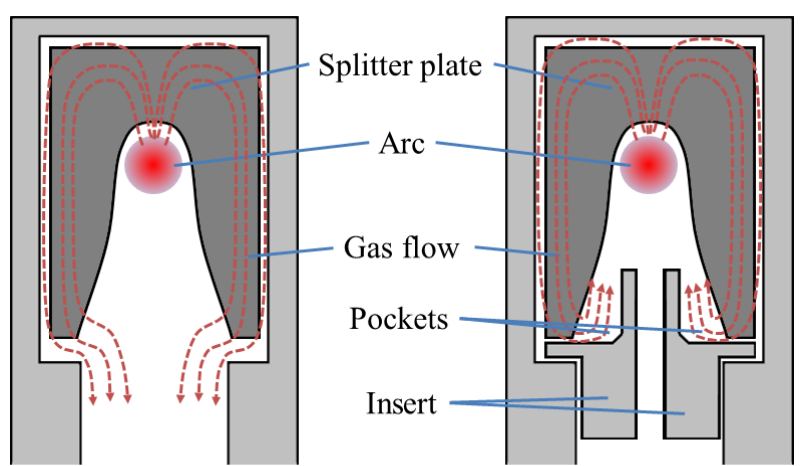

(a) Without insert

(b) With insert

Figure 5. Gas flow redirection using insert.

To determine the effectiveness of the device, shortcircuit testing with and without the insert have been performed. Some results are presented in Fig. 6. In the test without the insert, some droplet solidified and did stick below the contact arm. That led to an interference with the motion of the contact arm. Using the insert, the debris issues could successfully be reduced and the tests were successful.

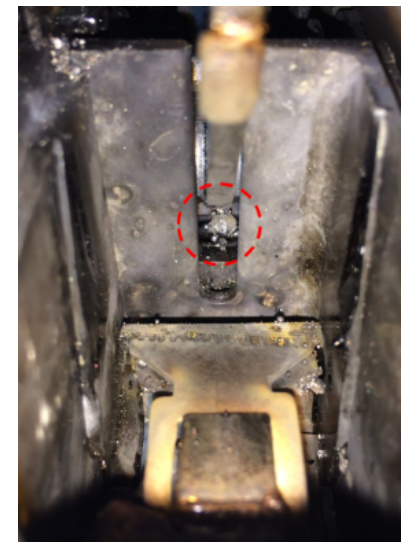

(a) Without insert

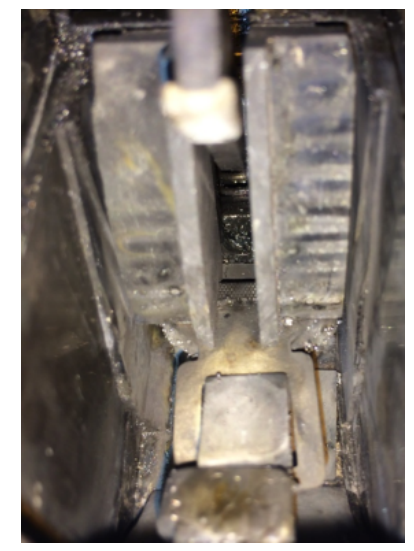

(b) With insert
Figure 6. View inside arc chambers of circuit breakers after testing (cover and stack of splitter plates removed). 


\section{Application: Cooling device for MCCB exhaust venting}

\subsection{Introduction}

During short-circuit interruption (SC), low-voltage circuit breakers vent hot plasma gas into the surroundings. To evaluate the potential harm of these gases, a metal mesh is placed around the breaker during SC testing according to IEC standard 60947-2 [6]. This metal mesh is connected to the ground via a fuse. If the vented gasses are still ionized (conductive) and the distance to the metal mesh is too short, current can flow from the breaker over the metal mesh to ground, eventually blowing the fuse. In this case the test would have failed.

To reduce this risk, a novel approach based on a heat exchanger device that is attached to the venting ports of the breaker was developed. By means of a larger number of thin metal plates with small gaps in between, the gas flow is cooled very effectively without changing the interruption performance of the breaker. The goal is to cool down the gases to a safe temperature level, so that even the risk of breakdown between conductive parts external of the breaker is eliminated.

\subsection{Arc model}

To enable the dimensioning of the cooling device, arc modeling is used to determine the venting conditions of the circuit breaker. An MCCB with rated current of $630 \mathrm{~A}$ has been investigated in this study that is based on a single-break design. The 3D MHD arc modeling approach, as introduced in Sec. 2, is used to calculate the AC short-circuit interruption behavior for $35 \mathrm{kA}$ prospective current at line voltage of $600 \mathrm{~V}$. One half of one pole of the breaker is modeled, applying a symmetry boundary condition at the center of the pole. Fig. 7 shows the temperature distribution inside the arc chamber at $t=2.8 \mathrm{~ms}$ (symmetry plane as cut through the center of one pole). An iso-surface of temperature at $12000 \mathrm{~K}$ is used to visualize the arc shape. The electrical network sub model is used to describe the interaction of the arc with the electrical network that is connected to the modeled device. Typical venting parameters like temperature and mass flow have been extracted from the model results.

\subsection{Dimensioning of the cooling device}

Modeling methods have been applied to determine optimal design parameters of the heat exchanger device. As a first step, the optimization goal needs to be determined. To avoid a breakdown between conductive parts that are in the venting path outside the breaker (e.g. live busbars), a safe venting gas temperature level has to be determined since the breakdown probability increases with higher venting gas temperatures, as shown by Tanaka [8]. The reduced critical electric field strength $\left(\frac{E}{N}\right)_{\text {cr }}$ of air and air-copper mixtures decreases with increasing temperature, but up to $1500 \mathrm{~K}$

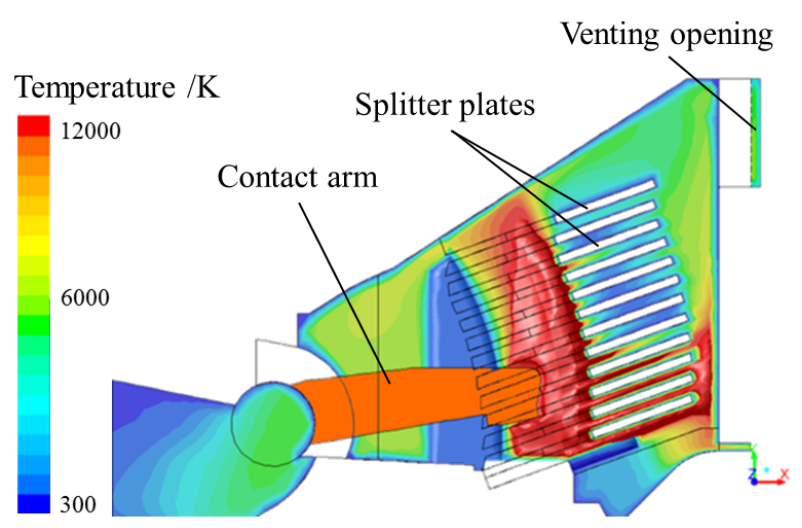

Figure 7. Arc simulation model result ( $t=2.8 \mathrm{~ms})$.

the reduced critical electric field strength remains almost at the same level. Since the particle density $N$ is decreasing with rising temperature, the critical electric field $E_{\text {cr }}$ is decreased by a factor of 5 when increasing the temperature from $300 \mathrm{~K}$ to $1500 \mathrm{~K}$. The critical field of air then drops from $30 \mathrm{kV} / \mathrm{cm}$ to about $6 \mathrm{kV} / \mathrm{cm}$ (at atmospheric pressure). Metal vapor contamination does not play a role in this case, because at $1500 \mathrm{~K}$ all potential metal vapor that is coming from the arc chamber should be condensed. Liquid or condensed metal particles can influence the dielectric strength, but these effects are outside the scope of this study. Still some plastic decomposition products coming from the arc interaction with plastic materials - could have an influence at this temperature level. Narayanan et al. [9] calculated the critical electric field for different air-copper-PA66 mixtures. Using these results, a value of about $2.5 \mathrm{kV} / \mathrm{cm}$ could be derived for the critical electric field.

With some typical dimensions, one can derive an approximate temperature threshold. The minimal clearance in air according to IEC standard 61439-1 [10] is $8 \mathrm{~mm}$ for rated impulse withstand voltage of $8 \mathrm{kV}$. Assuming that conductors have radii of $2 \mathrm{~mm}$ and the applied voltage is up to $1000 \mathrm{~V}$, the electric field has approximately a maximum value of $2 \mathrm{kV} / \mathrm{cm}$ (uniform electric field is $1.25 \mathrm{kV} / \mathrm{cm}$ ). This is still below the critical field value of $2.5 \mathrm{kV}$ as shown above, so one can conclude that $1500 \mathrm{~K}$ is a good estimation for a safe venting gas temperature, considering that any venting gas will mix with cold gas outside the breaker reducing the gas temperature further.

The second optimization goal refers to the interruption performance of the breaker. The pressure drop created by the additional cooling device should be minimal. That ensures that the arc motion inside the breaker is not affected by the additional cooling device compared with the original breaker.

Instead of building many prototypes and running tests, a numerical "Design of Experiments" (DoE) study was performed using a simplified 2D model as shown in Fig. 8. With two symmetry boundary conditions defined, the model represents a cooling 


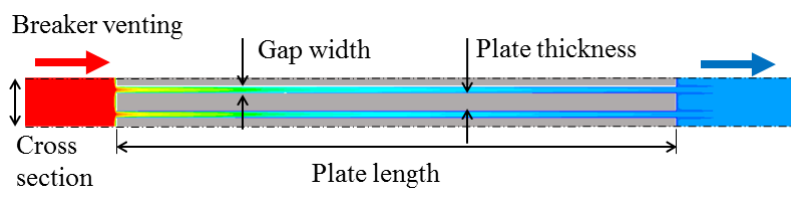

Figure 8. Simplified 2D model used in DoE study of the cooling device.

plate in the center, two adjacent gaps, and half of the two adjacent plates. Following design parameters describe the configuration: cross section, gap width, plate thickness, and plate length. Using mass-flow and temperature values as determined in the $3 \mathrm{D}$ arc simulation, a full-factorial DoE with center point is performed and evaluated where the design parameters serve as factors. Results are shown in Fig. 9, representing the normalized effects plot (ratio of effect to maximum effect) for each factor as an indicator, which design parameter or parameter combination has the most impact on the cooling device performance. To reduce the gas temperature with limited addition of pressure drop, a large total cross section is the most important design parameter. Reduction of the width of the gap between the plates also determines the cooling efficiency, but also increases the pressure drop (at fixed cross section). The length of the device can be used to control the cooling rate without changing the pressure drop, since most of the pressure drop occurs in the first section of the device.

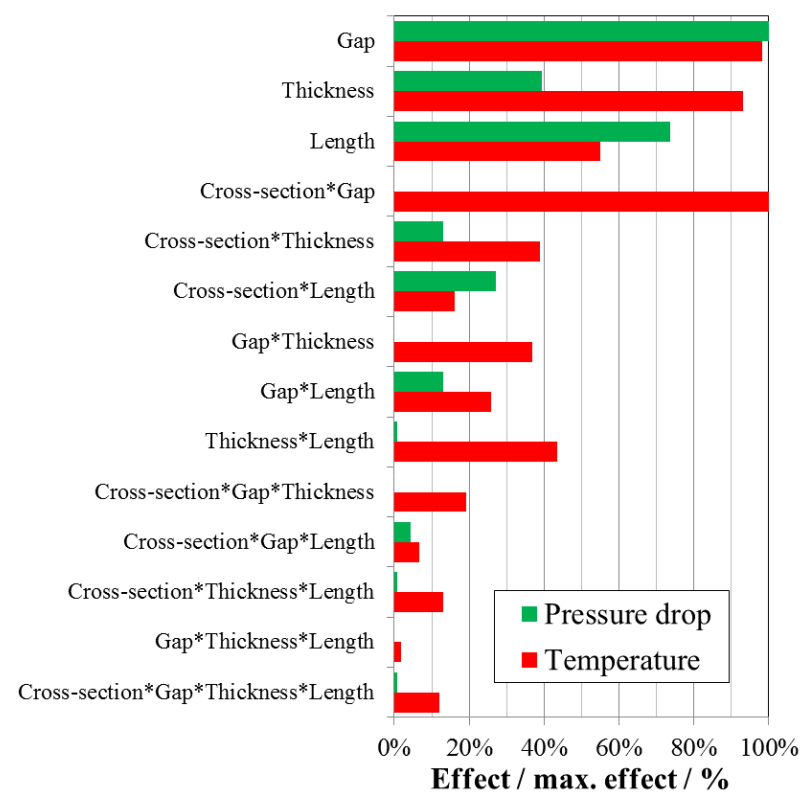

Figure 9. DoE study results for blow-out cooling.

\subsection{Results}

Based on the DoE study results, some prototypes with different dimensions were built and tested. In Fig. 10 a design employing 55 plates per pole with a thickness of $0.4 \mathrm{~mm}$ and gap of $0.2 \mathrm{~mm}$ is shown.

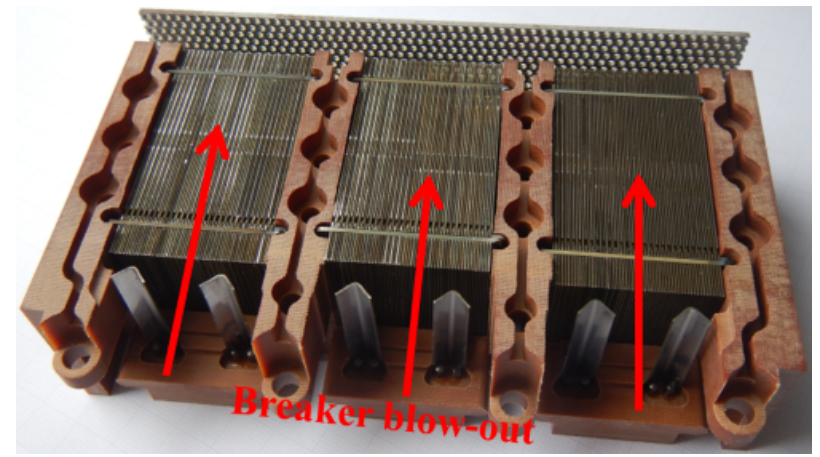

Figure 10. Cooling device (cover removed).

Short-circuit testing was done proving that the two performance goals can be achieved. Fig. 11 shows single pole interruption testing results for $35 \mathrm{kA}$ prospective current at $600 \mathrm{~V}$, comparing the breaker behavior with and without cooling device. Although the test conditions are not $100 \%$ identical (tests with and without cooling device at $50 \mathrm{~Hz}$ and $60 \mathrm{~Hz}$ circuit frequencies respectively), the interruption is very comparable. The arc voltage behaves almost identical, therefore one can conclude that the arc motion is not influenced by the cooling device.

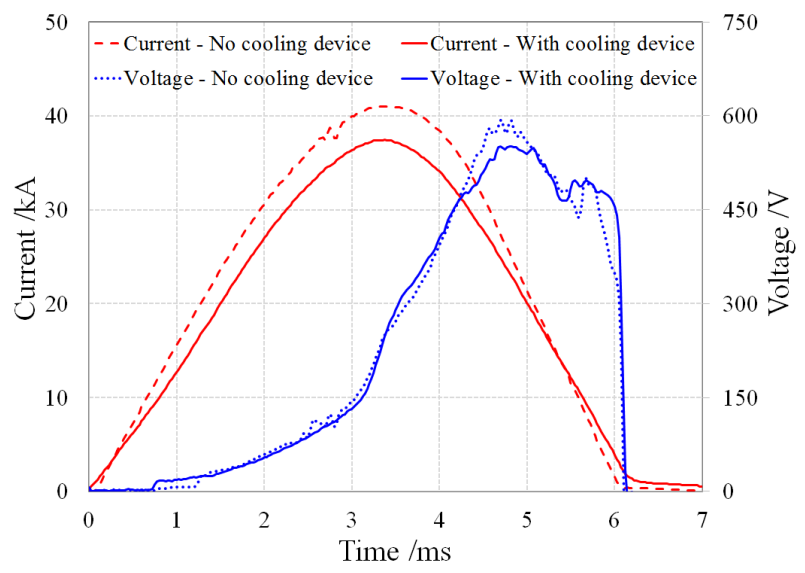

Figure 11. Test results for $35 \mathrm{kA}$ single phase interruption, without and with cooling device.

To detect the cooling effect, a breakdown detection test setup is mounted external to the breaker directly into the venting path. It consists of a several pairs of metal rods with $8 \mathrm{~mm}$ distance and $2 \mathrm{~mm}$ radius, placed at four different distances away from the venting port of the breaker or the attached cooling device. Closest achievable distance of the first rod pair to the venting port in the setup was $10 \mathrm{~mm}$. A capacitor was pre-charged to $853 \mathrm{~V}$ (slightly above $\sqrt{2} 600 \mathrm{~V}$ ) and connected to the metal rods. If the gas stream coming from the breaker - directly or from the cooling device - is passing over the metal rods, a potential breakdown through the $8 \mathrm{~mm}$ gap can be detected while monitoring the voltage level. If the gas is still conductive or in case of a breakdown, the capacitor will discharge and the voltage collapses. 
With aid of this setup, the risk mitigation using the cooling device could be demonstrated. In 18 different test with 72 different measured potential breakdown positions, only a single breakdown could be detected. Analyzing the breaker, it was clear that some parasitic venting not flowing through the cooling device caused that single breakdown event.

\section{Application: Hollow core surge arrester}

\subsection{Introduction}

Surge arresters employing metal-oxide varistor elements (MOV's) are widely used to protect medium and high voltage electrical distribution equipment from over voltage transients caused by internal (switching) or external events (e.g. lightning strike). MOV elements act as an electrical insulator under normal operating conditions and become electrically conductive in case the line voltage exceeds the designed threshold, creating a conductive path to ground. A short-circuit (SC) event inside the surge arrester can occur if the energy that needs to be dissipated is exceeding its capability. Surge arresters must be designed and tested as per IEC/IEEE standards for short-circuit faults to protect surrounding personnel and equipment.

In this section "Design A"type arresters according to IEC60099-4 [11] are investigated. This arrester design typically consists of metal-oxide varistor blocks that are stacked inside a hollow fiberglass reinforced polymer tube, as shown in Fig. 12. The silicone rubber housing (UltraSil ${ }^{\mathrm{TM}}$ ) around the tube enhances the electrical isolation even under extreme environmental conditions. The tube is mounted onto two heavy duty aluminum end castings that provide secure mounting provisions. Cast in horns (nozzles), as part of the end castings, enable directional venting of hot gases in case the built-in pressure relief device is activated due to an end-of-life event. Besides the pressure-relief functionality, the metal diaphragm as shown in Fig. 12, provides sealing of the MOV's from the environment.

In the event of a short-circuit inside the tube, a high current is flowing in a confined space forming a hot ionized plasma column inside the arrester. Heat transfer mechanisms radiation, convection, and conduction are distributing the arc energy inside the arrester cavities. Electromagnetic forces, acting on the plasma column, are responsible for further distribution of the hot ionized gas inside the tube. This results in fast heating of the air inside the surge arrester tube and a fast pressure rise that triggers the rupture of the diaphragms, once a pressure threshold is exceeded. From that point onwards hot ionized gas is venting through the nozzles to the exterior of the tube.

However, as current continues to flow through the interior of the tube, the tube pressure continues to rise gradually and drives venting of the hot gas through the nozzles. At some point in time the streams of ionized gas from top and bottom nozzles will meet and the arc

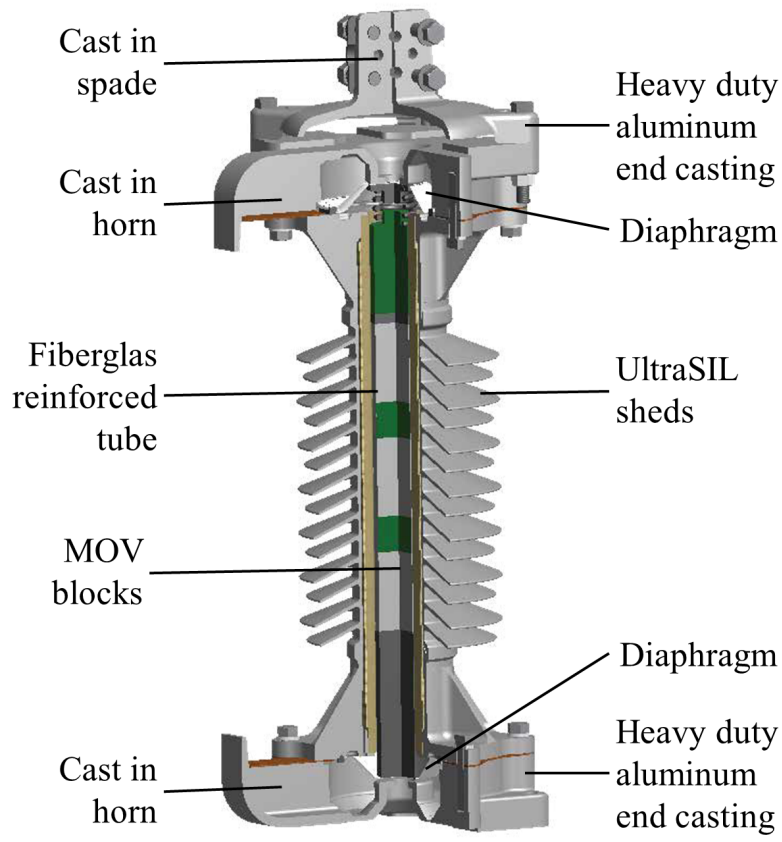

Figure 12. Principle design of a VariSTAR ${ }^{T M} H C$ station class surge arrester.

is commutating to the exterior region. With the arc burning outside the arrester, the internal pressure rise is limited and therefore the thermal and mechanical stresses on the tube are also limited, as reported by Ozawa et al. [12]. With the help of high speed imaging, the external arc commutation phenomenon is analyzed in the SC test and compared with gas flow analysis. It was shown that a quick arc commutation from inside to outside is a key to limit pressure inside surge arrester [12].

In the certification test according to IEC60099-4 [11] a fault condition is initiated using a fuse wire that is placed in the cavity inside the tube. Testing is carried out for high, intermediate and low short-circuit currents and the arrester must satisfy various criteria in order to pass the tests. In short, no violent shattering of the structure is permitted where parts or fragments of more than $60 \mathrm{~g}$ leave the device during testing. The Arrester shall be able to self-extinguish open flames within two minutes at the end of the test.

Since prototyping and SC testing of surge arresters is tedious and expensive, a simulation model is developed to enhance the understanding of the physical phenomena during interruption to be able to reduce the development time.

\subsection{Model approach}

The 3D coupled multi-physics model approach introduced in Sec. 2 is applied to the surge arrester geometry. The simulation domain represents the interior of the arrester as well as some air volume outside the device to be able to capture arc commutation events. Due to the high energy density in the tube during short-circuit testing, high pressure values are expected 
and some deformation of the device will happen in reality. The model does not reflect the effect of tube deformation, although the effect of the pressure relief due to the diaphragm is captured once a pressure threshold is exceeded.

Calculations are done to represent and analyze three different load scenarios where test results are available (with peak current values of $I_{\mathrm{p}}=82.5 \mathrm{kA}, 98.6 \mathrm{kA}$, and $126 \mathrm{kA}$ ) with focus on the initial time period where the arc commutation is happening (full test duration of $200 \mathrm{~ms}$ is not modeled). The current traces recorded during testing were used as load in the model to calculate the flow and electromagnetic processes. Since the complex phenomena that occur during melting and vaporization of the fuse wire are out of scope of the model, the initial condition is defined by patching a small hot arc channel inside the surge arrester where the fuse wire would be located. This enables the start of the calculation run, but the time point that is represented by the model compared to the real test needs to be determined. The arc voltage trace is used to define the time offset for the simulation, because the rapid decay of the measured voltage during the initial time period indicates the vaporization of the fuse wire and establishment of an arc discharge in the tube (see Fig. 13, Fig. 14, and Fig. 15).

\subsection{Model results}

Arc voltage and arc commutation time for $I_{\mathrm{p}}=82.5 \mathrm{kA}$, $98.6 \mathrm{kA}$, and $126 \mathrm{kA}$ peak current tests are compared with simulation data in Fig. 13, Fig. 14, and Fig. 15 respectively. As indicated before, the predicted arc voltage deviates from the measurement in the initial time period due to the initial model settings and assumptions.

In the the first load case with $I_{\mathrm{p}}=82.5 \mathrm{kA}$, as shown in Fig. 13, the arrester tube ruptured in the test before the arc could commutate from the interior to the outside. Hot ionized gas is leaking at the middle section of the tube, as seen in the picture in Fig. 13 early in the test. The hot gas does help to form a conductive path outside the tube much faster compared to the case where the tube remains intact and the conductive channel is formed by the nozzle gas streams only. Therefore the arc voltage does decrease early on in the test. As the model cannot consider the tube rupture, the predicted arc voltage deviates from test result showing a later arc commutation at about $3.8 \mathrm{~ms}$.

The second load case with $I_{\mathrm{p}}=98.6 \mathrm{kA}$ is shown in Fig. 14. In this test the tube ruptured as well, but the arc did commutate after the rupture occurred. In this case the arc voltage almost remains constant due to gradual expansion of plasma inside pressurized arrester tube. During arc commutation, two hot gas streams meet outside to create a low resistance path for current flow, resulting in sudden drop in arc voltage. It should be emphasized that the tube rupture does not mean the test failed, as long as test criteria as per

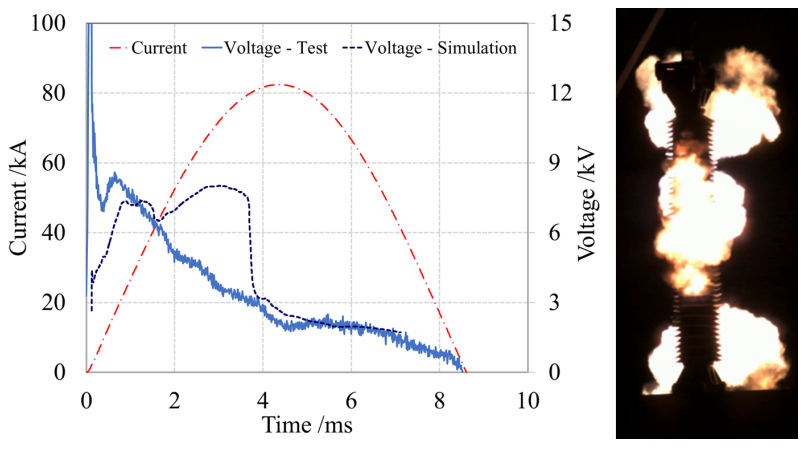

Figure 13. $I_{p}=82.5 \mathrm{kA}$ peak current, tube ruptured before arc commutation. Picture taken at $1.12 \mathrm{~ms}$.

the standard are satisfied. The model does predict this commutation $1 \mathrm{~ms}$ earlier compared to the test. One explanation is some additional venting that was observed in the test, lowering the tube pressurization. As this venting is not considered in the model, a higher pressure inside the tube is predicted and the gases are accelerated faster through nozzle leading to an earlier arc commutation in the model.

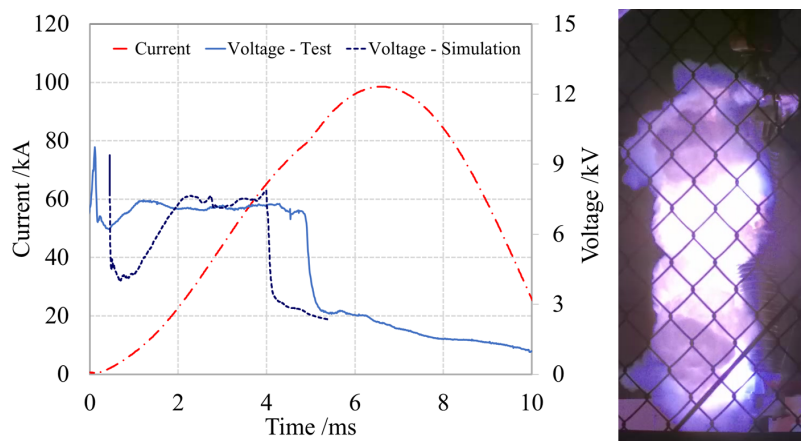

Figure $14 . \quad I_{p}=98.6 \mathrm{kA}$ peak current, tube ruptured after arc commutation. Picture taken at $4.95 \mathrm{~ms}$.

In the third load case with $I_{\mathrm{p}}=126 \mathrm{kA}$, as shown in Fig. 15, no indication for additional venting was found, also the tube did remain intact. Thus the simulation model mimics the test conditions closely and the predicted arc commutation time of $4.8 \mathrm{~ms}$ is in good agreement with the test result. But another effect is now visible that seems to lead to deviations of the predicted arc voltage as seen by the calculated over- and undershooting voltage results.

The interior air volume inside the surge arrester is divided into a number of channels along the length by some baffles. Depending on the current rise, the model predicts a different distribution of the arc plasma inside the tube where the available space might be more or less effectively utilized. This does lead in the $I_{\mathrm{p}}=126 \mathrm{kA}$ case to a over and undershoot of the arc voltage, which can be seen in the other cases as well but not as clearly. In the test this does not seem to happen because the tube baffles might have deformed, providing more area for the arc to expand into some adjacent channels. This could be confirmed by some numerical test with bigger baffle gaps, leading to a 


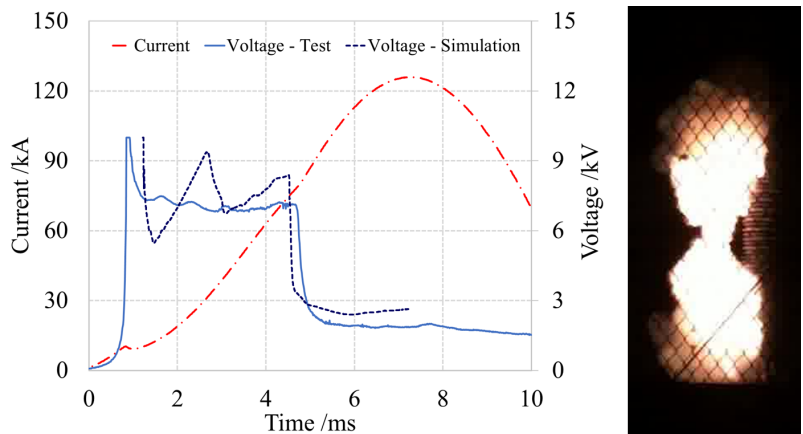

Figure 15. $I_{p}=126 \mathrm{kA}$ peak current, tube not ruptured. Picture taken at $4.82 \mathrm{~ms}$.

flat arc voltage result (not shown here).

As discussed, the arc commutation phenomenon is recorded with a high speed camera and shown for some time points in Fig. 16. For the same time points the simulation results are shown as iso-surfaces of temperature and compared with test images for different time points in Fig. 16. The expansion of the plasma plum, as indicated by the temperature iso-values at $5000 \mathrm{~K}$ and $10000 \mathrm{~K}$, does correspond well with the expansion during the test.
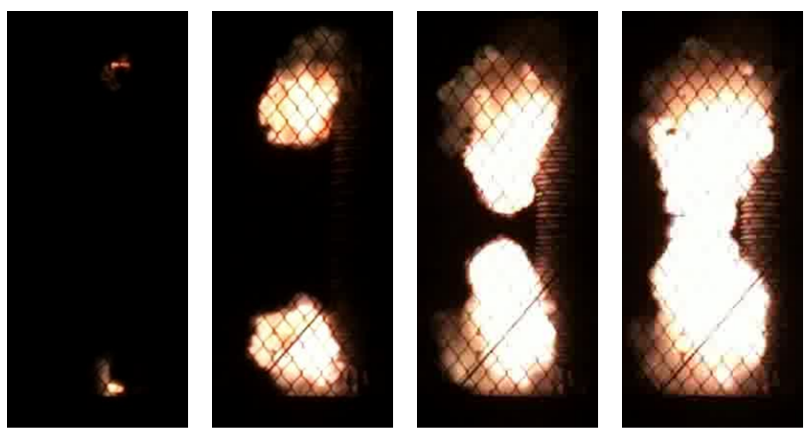

(a)

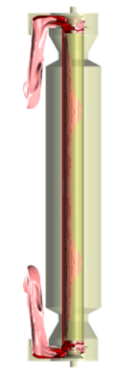

(b)

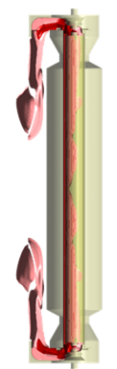

(c)

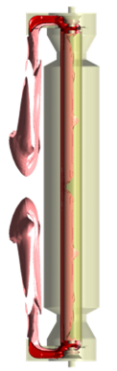

(d)

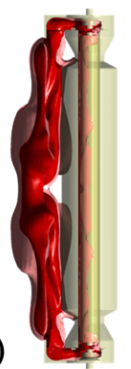

Figure 16. Arc commutation process in experiment and simulation. Images from $H S$ video for different time points of $I_{p}=126 \mathrm{kA}$ fault case: (a) $3.00 \mathrm{~ms}$, (b) $3.5 \mathrm{~ms}$, (c) $4.29 \mathrm{~ms}$, and (d) $4.88 \mathrm{~ms}$. Iso-surfaces of $5000 \mathrm{~K}$ and $10000 \mathrm{~K}$ shown to visualize the expanding plasma jets.

\section{Conclusions}

By means of three industrial application examples we discussed the utilization of arc modeling methods in this contribution. Although not all complex physical processes or interactions can be included in the model yet, the model results did provide useful insights for better understanding of the designs and to be a useful aid in the design process. In particular, the particle tracking results for the MCCB short-circuit application lead to the new debris catching insert design that significantly improved the short-circuit performance at high prospective current values. In case of the blow-out cooling device, a numerical DoE study did help to determine the optimal design parameters. The effectiveness of the design has been proven by various tests. In the high voltage surge arrester application, arc modeling did lead to a better understanding of the arc commutation process, which can be used in future to optimize the design further.

\section{References}

[1] A. Gleizes, J. J. Gonzalez, and P. Freton. Thermal plasma modelling. Journal of Physics D: Applied Physics, 38(9):R153, 2005. doi:10.1088/0022-3727/38/9/R01.

[2] C. Rümpler. Lichtbogensimulation für

Niederspannungsschaltgeräte. Dissertation, Technische Universität Ilmenau, 2009.

[3] ANSYS ${ }^{\circledR}$ Fluent and ANSYS ${ }^{\circledR}$ Emag. Release 19.1, ANSYS, Inc., 2018.

[4] MpCCI ${ }^{\circledR}$-E Manual. 2018, Fraunhofer Institute SCAI, Sankt Augustin, 2018.

[5] C. Rümpler, H. Stammberger, and A. Zacharias. Low-voltage arc simulation with out-gassing polymers. In 2011 IEEE 57th Holm Conference on Electrical Contacts (Holm), pages 1-8, Sept 2011. doi: 10.1109/HOLM. 2011.6034770.

[6] Low-voltage switchgear and controlgear - Part 2: Circuit-breakers. IEC Standard 60947-2, The International Electrotechnical Commission, 2016.

[7] C. Rümpler, B. J. Schaltenbrand, A. L. Gottschalk, R. P. Malingowski, K. J. McCarthy, and X. Zhou. Electrical switching apparatus and debris barrier therefor. U.S. Patent 10128 069, Nov 2018.

[8] Y. Tanaka. Influence of copper vapor contamination on dielectric properties of hot air at $300-3500 \mathrm{~K}$ in atmospheric pressure. IEEE Transactions on Dielectrics and Electrical Insulation, 12(3):504-512, June 2005. doi:10.1109/TDEI.2005.1453455.

[9] V. R. T. Narayanan, J. V. R. Heberlein, and C. Rümpler. The influence of metallic and plastic vapors on dielectric breakdown for low-voltage circuit breaker after current-zero. In 2013 IEEE 59th Holm Conference on Electrical Contacts (Holm 2013), pages 313-318, Sep 2013. doi:10.1109/HOLM. 2013.6651435.

[10] Low-voltage switchgear and controlgear assemblies Part 1: General rules. IEC Standard 61439-1, The International Electrotechnical Commission, 2011.

[11] Surge arresters - Part 4: Metal-oxide surge arresters without gaps for a.c. systems. IEC Standard 60099-4, The International Electrotechnical Commission, 2014.

[12] J. Ozawa, A. Mizukoshi, S. Maruyama, K. Nakano, K. Saito, G. St-Jean, Y. Latour, and A. Petit. Pressure relief design and performance of metal oxide surge arresters. IEEE Transactions on Power Delivery, 1(1):151-156, Jan 1986.

doi: 10.1109/TPWRD . 1986. 4307901. 